\title{
VIII.
}

Aus dem pharmakologischen Institut au Marburg.

\section{Zur Theorie der Alkoholnarkose.}

Zweite Mittheilung.

Ein physikaliseh-chemiseher Beitrag zur Theorie der Nareotica.

Von

Dr. Fritz Baum.

Nach der in dem vorangebenden Aufsatz von Professor Hans Meyer entwiekelten Theorie soll die Wirkungsstärke der aliphatischen Nareotica eine Funktion des Theilungseoëffieienten sein, nach dem sie sich im ganzen Organismus und in der Protoplasmaemulsion der Zellen zwisehen wässeriger Lösung und fettartigen Stoffen (Protagonen u. s. w.) physikaliseh vertheilen.

Um diese Annahme zu prüfen, war es zunächst erforderlieh, nach einem brauchbaren Maasse die Wirkungsstärke versehiedener Nareotica festzustellen. Aeltere Beobachtungen wie auch neuere Studien, z. B. von Houdaille ${ }^{1}$ ) und von Chabriés) waren fur den vorliegenden Zweck nioht ohne weiteres zu verwerthen, weil sie als Maass der Wirkungsstärke die tödtliche Maximaldosis oder wie Chabrié das Product ans der jeweils tödtlichen Dosis in die Zeit bis zum Wintritt des Todes aufstellten; der Tod ist aber keineswegs immer die Folge gleiehartiger und für die hier vorliegende Frage in Betracht kommender, sondern oft ganz nebensäehlicher Wirkungen,

1) Houdaille, Étude expérimentale et critique sur les nouveaux Hypnotiques. Thèse Paris 1893.

2) Chabrié, Compt. rend. Acad. d. Sciences. Paris 1893. 
und es bedarf nicht weiterer Ausführung, dass die Narkose selbst, d. h. der Ausfall bestimmter, leicht erkennbarer Funktionen des Centralnervensystems als Index zu benutzen sein wird. In dieser Weise sind für unseren Zweck von Diehli), Dunzelt') u. z. Th. von $\mathrm{Bucholz} \mathrm{z}^{3}$ ) an einer grösseren Reihe von narkotischen Stoffen vergleichende Versuche angestellt worden: als Versuchsthiere dienten hauptsächlich Frosehlarven, bei denen ein bestimmter Grad der Narkose sich an dem Aufhören gewisser Reflexe genügend scharf erkennen liess. In ähnlicher Weise hat dann auch Juekuff ${ }^{4}$ ) an kleinen Fischen, ihre respiratorische Kiemenbewegung als. Index benutzend, vergleichende Versuche gemacht. Wenn man auf diese Art die jeweilige geringste moleculare Concentration der einzelnen Nareotica bestimmt, die eben noch im stande ist, die zu beobachtende Narkosenwirkung herbeizuführen, so gelangt man zu Grenzoder Schwellenwerthen, die sich vergleichen und auf Grund deren sich die Narcotica in eine Stärkeskala bringen lassen.

Dass eine solche theoretisch annähernd richtige Skala nicht auf die entsprechenden Wirkungen an Vögeln, Säugethieren $u$. s. w. übertragen werd̃en dürfe, ist einleuchtend, wenn die verschiedene Löslichkeit, Diffusions- und Resorptionsfähigkeit, sowie die Zersetzlichkeit der einzelners Narcotica berücksichtigt wird. In betreff der Versuche von $J$ offroy und Serveaux ${ }^{5}$, diese Fehlerquellen bei Sängethierexperimenten zu vermeiden, verweise ich auf die Besprechung bei $D$ unzelt.

Iob gebe hier eine aus den vorstehend genannten Untersuchungen sich ergebende, der Stärke nach absteigende Reihe. Die beigesetzten Schwellenwerthe S sind ansgedrückt in Bruchtheilen der Normallösung (1 Grammmolecuil auf 1 Liter) und stellen selbstrerständlich nur Näherungswerthe dar:

1) Diehl, Vergleichende Experimentaluntersuchungen über die Stärke der narkotischen Wirkung einiger Sulfone, Säureamide und Glycerinderivate. Dissert. Marburg 1894.

2) Dunzelt, Vergleichende Experimentaluntersuchungen über die Stärke der Wirkung einiger Narcotica. Dissert. Marburg 1896.

3) Bucholz, Beiträge zur Theorie der Alkoholwirkung. Dissert. Marburg 1895 .

4) Juckuff, Versuche zur Auffindung eines Dosirungsgesetzes. 1895. Analoge und zum Theil gleichmässigere Resultate lieferten $\mathrm{Ju} \mathrm{cku}$ ff seine entsprechenden Beobachtangen über die Auflösung von rothen Blutkörperchen; doch bedarf es dazu so starker Concentrationen der betr. Narcotica, dass es bei sehwer löslichen, wie Sulfonal, überhaupt nicht zur Hämatolyse kommt.

5) Joffroy und Serveanx, Considérations générales sur la recherche de la toxicité. Arch. de Méd. expér. et d'anat. path. 1896. 
S.

$\begin{array}{ll}\text { Tetronal } & 0,0013 \\ \text { Trional } & 0,0018 \\ \text { Butylchloralhydrat } & 0,0020 \\ \text { Bromalhydrat } & 0,002 \\ \text { Dichlorhydrin } & 0,002 \\ \text { Chloralose } & 0,004 \\ \text { Sulfonal } & 0,006 \\ \text { Triacetin } & 0,010^{1} \text { ) } \\ \text { Diacetin } & 0,015 \text { ) } \\ \text { Chloralhydrat } & 0,02 \\ \text { Aethylidenurethan } & 0,02 \\ \text { Chloralamid } & 0,04 \\ \text { Aethylurethan } & \mathbf{0 , 0 4} \\ \text { Glycerinäther } & 0,04 \\ \text { Monochlorhydrin } & 0,04 \\ \text { Monacetin } & 0,051) \\ \text { Propylenglykol } & 0,2 \\ \text { Methylurethan } & 0,4 .\end{array}$

In 2. Linie wäre nun mit dieser Scala die Reihe der entsprechenden Theilungscoëffieienten zu vergleichen, von denen eine grössere Anzahl festzustellen ich im Folgenden unternommen habe.

Ich bin von der vorläufigen Annahme ausgegangen, dass in Bezug auf ihre mechanischen Affinitäten - in specie Lösung der Narcotica - die fettartigen Zellsubstanzen, Protagone, Lecithine u. s. w. sich im grossen und ganzen den einfachen Fetten ähnlich verhalten, wenigstens im Vergleich und Gegensatz zu Wasser und wässerigen Lösungen; ich habe also die Vertheilung der Narcotica in einem Gemenge von $\mathrm{W}$ asser und Olivenöl bestimmt.

Da die Lösliehkeit von Aether, Chloroform u. s. w. in Wasser and in Oel durch die Temperatur ungleich beeinflusst wird, so ist auch der Theilungseoëfficient von der Temperatur des Wasser-Oelgemisches abhängig. Ich bin indes auf diese von wechselnder Temperatur bedingten aber meist in gleichem Sinne verlaufenden Abweichungen der Theilungseoëfficienten nicht eingegangen, sondern habe zunächst die Versuche bei gleichmässiger Zimmertemperatur $\left(18^{\circ} \mathrm{C}\right.$.) angestellt.

Das Ergebniss ist die nachstehende Reihe mit danebengesetzten Theilungscoëfficienten $\frac{\mathrm{Cf}_{f}}{\mathrm{C}_{\mathrm{W}}}{ }^{2}$ und Schwellenwerthen $\mathrm{S}$.

1) Indirect abgeleitete Werthe.

2) $C_{f}$ und $C_{w}$ die Concentration in Fett, bezw. Wasser. 


\begin{tabular}{|c|c|c|c|c|c|}
\hline & & & & $\frac{\mathrm{C}_{\mathfrak{f}}}{\mathrm{C}_{\mathrm{W}}}$ & $S$ \\
\hline Trional . . & . . & •.. & . & 4,46 & 0,0018 \\
\hline Tetronal. . & . . & - . & . & 4,04 & 0,0013 \\
\hline Butylchloralhydx & Irat . & - . & . & $1, \tilde{5} 9$ & 0,0020 \\
\hline Sulfonal . & . . & $\cdot$. & . & 1,11 & 0,0060 \\
\hline Bromalbydrat & .. & . . & . & 0,66 & 0,0020 \\
\hline Triacetin . & . . &. & . & 0,30 & 0,010 \\
\hline Diacetin . . & . . & . . & . & 0,23 & 0,015 \\
\hline Chloralhydrat & . . & $\therefore$. & . & 0,22 & 0,020 \\
\hline Aethylurethan & - & . & . & 0,14 & 0,040 \\
\hline Monacetin . & . & . & - & 0,06 & 0,050 \\
\hline Methylurethan & . . & . . & . & 0,04 & 0,40 \\
\hline
\end{tabular}

Die Uebereinstimmung in der Reihenfolge ist, wie man sieht, nahezu vollständig und bei der unvermeidlichen Ungenauigkeit in der Bestimmung der Wirkungsstärke und mitunter auch des Theilungscoëfficienten kaum besser zu erwarten.

Zur weiteren Prüfung des Zusammenhanges zwisehen Wirkungsstärke und Theilungscoëfficient wurde letzterer noch fur zwei zu den Sulfonen gehörigen Verbindungen und für den tertiären Butylund Amylalkohol ermittelt. Die Prüfung der narkotischen Wirkung der beiden ersten Substanzen war durch Baumann und Kasti) bei ihrer eingehenden Untersuchung über die Sulfone geschehen. Sie hatten dabei gefunden, dass nur diejenigen Verbindungen wirksam seien, welche eine Aethylgruppe enthalten und beim Durehgang: durch den Organismus zersetzt werden. Daraus schien gefolgert werden zu dürfen, dass der Aethylgruppe für das Zustandekommen der narkotischen Wirkung ein besondere Bedeutung zukomme. Diese Annahme glaubten v. Mering und Schneegans 1) in einer Untersuchung über die Wirkung verschiedener Alkohole bestätigt zu finden, da sich die an einem tertiären Kohlenstoffatom eine Aethylgruppe enthaltenden Verbindungen gegenüber denjenigen, welehe dafür eine Methylgruppe besitzen, als viel stärker wirksam erwiesen.

Von den Sulfonen wählte ich einerseits das Dimethylmethandimethylsulfon, $\left(\mathrm{CH}_{3}\right)_{2} \cdot \mathrm{C} \cdot\left(\mathrm{SO}_{2} \mathrm{CH}_{3}\right)_{2}$, weil es als eklatantes Beispiel für den Einfluss der Aethylgruppe dienen soll, da es, dem Sufonal (Dimethylmethandiäthylsulfon) ganz analog constituirt, fast wirkungslos ist, trotzdem es im Körper zersetzt wird, anderseits das Diäthyl-

1) Baumann und Kast, Zeitschrift t. physiol. Chemie Bd. XIV. 1890.

2) v. Mering und Schneegans, Therap. Monatshefte, Juli 1892. 
sulfonmethan $\mathrm{CH}_{2} \cdot\left(\mathrm{SO}_{2} \mathrm{C}_{2} \mathrm{H}_{5}\right)_{2}$, das ebenso, trotzdem es Aethylgruppen enthält, unwirksam ist, aber den Organismus unzersetzt passirt. Eine Zusammenstellung der Theilungscoëfficienten dieser Verbindungen mit denen der sehon früher angeführten stark wirkenden Sulfone und derjenigen der beiden tertiären Alkohole giebt folgendes Bild, das wieder den Zusammenhang der Wirkungsstärke und des Theilungscoëfficienten erkennen lässt.

\begin{tabular}{|c|c|c|c|}
\hline Substanz & Chemisehe Constitution & $\begin{array}{l}\text { Wirkungs- } \\
\text { grad }\end{array}$ & $\begin{array}{l}\text { Theilungs- } \\
\text { evêffic. }\end{array}$ \\
\hline Diäthylsulfonmethan & $\mathrm{CH}_{2} \cdot\left(\mathrm{SO}_{2} \cdot \mathrm{C}_{2} \mathrm{H}_{5}\right)_{2}$ & sehr schwach & 0,1514 \\
\hline Dimetbylsulfondimethylmethan & $\left(\mathrm{CH}_{3}\right)_{2} \cdot \mathrm{C} \cdot\left(\mathrm{SO}_{2} \cdot \mathrm{CH}_{3}\right)_{2}$ & dito & 0,106 \\
\hline Sulfonal : . . . . . & $\left(\mathrm{CE}_{3}\right)_{2} \cdot \mathrm{C} \cdot\left(\mathrm{SO}_{2} \cdot \mathrm{C}_{2} \mathrm{H}_{5}\right)_{2}$ & stark & 1,115 \\
\hline Trional . . . . . & 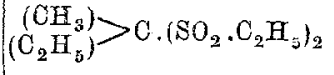 & $\begin{array}{l}\text { viel stärker } \\
\text { als Sulfonal }\end{array}$ & 4,458 \\
\hline Tetronal . . . . . & $\left(\mathrm{C}_{2} \mathrm{H}_{5}\right)_{2} \cdot \mathrm{C} \cdot\left(\mathrm{SO}_{2} \cdot \mathrm{C}_{2} \mathrm{H}_{5}\right)_{2}$ & dito & 4,039 \\
\hline tertiärer Butylalkohol . & $\left(\mathrm{CH}_{3}\right)_{3} \cdot \mathrm{C} \cdot(\mathrm{OH})$ & sehwaeh & 0,176 \\
\hline $\begin{array}{l}\text { tertiärer Amylalkohol } \\
\text { (Amylenhydrat) }\end{array}$ & $\left(\begin{array}{l}\left(\mathrm{OH}_{3}\right)_{2} \\
\left(\mathrm{C}_{2} \mathrm{H}_{5}\right)\end{array}\right\rangle \cdot \mathrm{C} \cdot(\mathrm{OH})$ & stark & 1,000 \\
\hline
\end{tabular}

Man ist nach diesen Zahlen zu dem Schlusse berechtigt, dass. die Aethylgruppe nicht die specifische Trägerin der narkotischen Wirkung ist, sondern dass lediglich die geänderten physikalisehen Verhältnisse die Stärke derselben beeinflussen, was unter Umständen ebensogut durch andere Gruppen, wie durch Acetyl bei den Acetinen, erfolgen kann. Eine Schwierigkeit scheint nur in dem eigenthümlichen Verhalten der verschiedenen Sulfone im Stoffwechsel zu liegen: indem nach Baumann's Angaben nur die im Organismus der Umwandlung unterliegenden Sulfone narkotisch wirksam sein sollen; wobei denn allerdings ihre physikalisehen Eigensehaften wohl bedeutungslos wären. Dagegen hat aber bereits Diehl es sehr wahrscheinlich gemacht, dass nicht die zersetzten, sondern die intacten Sulfone narkotisch wirksam seien: ich eitire den entsprechenden Satz aus seiner Arbeit:

Baumann und Kast behaupten: nur die im Organismus zersetzbaren Substanzen wirken. Dagegen sprechen meine Kaulquappenversuche, welche darthun, dass bei einigen Disulfonen die Wirkung fast momentan eintritt, obschon diese Körper chemisch sehr stabil und indifferent sind. Ferner ist dagegen einzuwenden, dass Kaulquappen, die lange Zeit - 24 Stunden und mehr - in einer Sulfonlösung narkotisirt gelegen haben, in reines Wasser gesetat nach wenigen Minuten erwachen. Es folgt daraus, dass das. in ihnen narkotiseh wirkende Gift von ihnen sehr schnell eliminirt oder vernichtet wird. Da die Narkose in der Sulfonlösung anhält. 
muss das eliminirte Gift immer wieder durch neu aufgenommenes ersetzt worden sein, d. h., wenn es sich um ein aus den Sulfonen entstehendes Zersetzungsproduct handelte, müsste nach kurzer Zeit die ganze Sulfonmenge oder ein merklicher Theil zersetzt worden sein. Man findet aber noch nach tagelangem Aufenthalt der Thiere in der Lösung die Masse des Sulfons nioht merklich verändert.

Ich selbst habe bei Gelegenheit von Untersuchungen über das Umwandlungsproduct des Trionals im Säugethierkörper, worüber ich später zu berichten gedenke, als vorlänfiges hier zu verwerthendes Resultat gefunden, dass die Ausscheidung des betreffenden Stoffwechselproductes mehrere Tage nach der Eingabe noch anhält, wann bereits jede narkotische Wirkung lange vorüber ist. Daraus ist zu schliessen, dass die chemische Veränderung der Sulfone nur ein secundärer, für das anfängliche Zustandekommen der Narkose gleichguiltiger Vorgang ist; als welcher aber vielleicht insofern damit znsammenhängt, als jene chemische Umwandlung gerade in den Zellen, die vermöge ihrer mechanischen Affinität die Sulfone fixiren, hauptsächlich geschehen könnte: daraus würde sich allenfalls erklären, warum die wirksamen Sulfone mit ihrem hohen Theilungscoëfficienten d. h. ihrer starken Affinität zu gewissen Zellprotoplasmen besonders energisch zerstört werden.

Baumann selbst hat übrigens beobachtet, dass solche Sulfone, die zwar die Aethylgruppen enthalten, die aber sehr leicht zerstört werden, ebenfalls wirkungslos seien: als solche führt er den Diäthylsulfonacetessigester und ein Homologes desselben an. Liegt in dieser. Bemerkung schon ein Widerspruch gegen die ganze $\mathrm{Bau}$ mann'sche Theorie, so steht sie dagegen in bestem Einklang mit der von uns vertretenen Anschauung, dass gerade das ungespaltene Sulfonmolecül das wirksame sei, und dass die Wirkung ausbleiben müsse, wenn die Spaltung zu rasch erfolgt: gerade so wie es bei den in der vorangehenden Mittheilung erwähnten leicht zersetzlichen Körpern, den Acetinen und Säureamiden, beobachtet ward; nach Application in den Magen trat die Wirkung äusserst schwach oder garnicht ein, nach Injection in die Blutbahn oder bei Versuchen an Kaltblütern erfolgte sie prompt.

Wennschon mein Versuchsmaterial kein grosses ist, und die gewonnenen Resultate noch weiterer und umfangreicherer Bestätigung bedürfen, so reichen sie doch aus, die ron Prof. H. Meyer aufgegestellte These sehr wahrscheinlich zu machen, dass die W ir kungsstäre der alkoholartigen Nareotica dureh ihren Theilungscoëficienten bedingt ist. 
Vor einiger Zeit hat Richet') es als ein Gesetz hingestellt, dass die Giftigkeit der Alkohole und Aether umgekehrt sich ververhalte wie ihre Löslichkeit in Wasser: "Plus ils sont solubles, moins ils sont toxiques." In dieser Form ist áber das vermeintliche Gesetz nicht richtig; man braucht nur an das in Wasser sehr leicht lösliche Chloral- und Bromalhydrat und an das schwer lösliche, aber ganz schwach wirksame Methylurethan zu denken; und auch unter ganz analog gebanten Körpern gilt die Regel nieht, denn das leichter lösliche Trional ist stärker wirksam als das weniger lösliche Sulfonal u. s. w. Riehtig wird das Gesetz aber, wenn mit dem Grad der Löslichkeit in Wasser der der Fettlöslichkeit gleichzeitig in Betracht gezogen wird, d. h. also der entsprechende Theilungscoëfficient. -

Nachdem ich versucht habe, den von der Theorie geforderten Zusammenhang an der Hand experimenteller Ergebnisse zu prüfen, will ich im Folgenden noch die Methodik meiner Versuche darstellen und kritisch besprechen.

Methoden und analytische Belege.

Nach den grundlegenden Untersuchungen von Berthelot und Jungfle isch ist der Theilungseoëfficient einer Substanz, d. i. das Verhältniss ihrer räumlichen Concentrationen bei der Vertheilung zwischen zwei sich miteinander nicht mischenden Lösungsmitteln, eine specifische Constante. Er ist von der Temperatur abhängig, unter bestimmten Umständen bei derselben Temperatur auch von der Concentration. Nach Nernst sind beide Lösungen im Gleichgewicht der Vertheilung isotonisch. Besitzt die Substanz in beiden Lösungsmitteln denselben Molecularzustand, so ist der Theilungscoëfficient von der Concentration unabhängig, bildet sie in einem derselben aber complexere Molecüle, wie beispielsweise die Essigsäure in Benzol, so muss in dieser Lösung die procentische Concentration in raseherem Verhältniss wachsen als in der anderen, damit die Lösungen isotonisch bleiben. Es ist in diesem Falle der Theilungscoëfficient von der Concentration abhängig. Diese Nernst'sche Auffassung dürfte dahin auszulegen sein, dass im Gleichgewicht der Vertheilung die osmotischen Drucke, nicht direct die Concentrationen, in einem constanten Verhältniss stehen. Handelte es sich einfach um isotonische Lösungen, so milssten alle Substanzen, welche in zwei bestimmten Lösungsmitteln denselben Molecularzustand besitzen, denselben Thei-

1) Richet, Compt. rend. Soc. Biol. Juli 1893. p. 775. Die ausführlichere Behandlung des Gegenstandes in $\mathrm{Houdaille,} \mathrm{Thèse} \mathrm{de} \mathrm{Paris} 1893$. 
lungscoëfficient haben. Diese Consequenz ist aber unvereinbar damit, dass diese für jede Substanz eine specifische Grösse ist. Es würde dabei ferner jeder Einfluss, welchen die Löslichkeit der Substanzen in den einzelnen Lösungsmitteln besitzt, unberücksichtigt bleiben, während es wahrscheinlich ist, dass das, was den Theilungscoëfficienten zu einer specifisehen Constanten stempelt, die Abhängigkeit desselben von diesen Löslichkeitsverhältnissen ist.

Zur Erkenntniss dieser Abhängigkeit scheint der Weg zu führen, welchen jüngst Stschukarew ${ }^{1}$ ) eingesehlagen hat. Er geht von Concentrationspaaren aus, welche sich im Gleichgewicht zu einander befinden. Ein solehes Concentrationspaar sind zunächst die beiden gesättigten Lösungen. 'Trägt man deren Concentrationswerthe als Abscisse und Ordinate in ein rechtwinkeliges Coordinatensystem ein, so erhält man einen Punkt der Curve, welche die Abhängigkeit des Theilungscoëfficienten vor der Concentration darstellt und für die Concentration Null durch den Ursprung des Coordinatensystems hindurchgeht. Stschukarew leitet einen Ausdruck ab, weloher aus den Werthen der gesättigten Lösungen und eines Concentrationspaares gebildet ist und für jedes beliebige der letzteren denselben Werth besitzt. Betreffs der Einzelheiten sei auf die Originalarbeit verwiesen.

Die Frage von der Abhängigkeit des Theilungscoëfficienten von der Concentration ist nach allem eine recht verwickelte und musste näher erörtert werden, weil darauf bei seiner experimentellen $\mathrm{Be}$ stimmung Rücksicht genommen werden muss. Ihrer Ausfïhrung möge folgende Ueberlegung zu Grunde gelegt werden. Ist der Gehalt eines beliebigen Volums $\checkmark$ der wässerigen Lösung vor dem Schütteln a, und nachher $b$, so entfällt die von demselben an das Oel abgegebene Substanzmenge $(a-b)$, wenn Wasser und Oel im Verhältniss $m: n$ zu einander stehen, auf das $\frac{\mathrm{n}}{\mathrm{m}}$ fache Volum, und die auf das gleiche Volum Oel v entfallende Menge ist, da

$$
\frac{\mathrm{n}}{\mathrm{m}} \mathrm{v}: \mathrm{v}=(\mathrm{a}-\mathrm{b}): \mathrm{x} \text {, }
$$

$\frac{\mathrm{m}}{\mathrm{n}}(\mathrm{a}-\mathrm{b})$. Das Verhältniss der in den gleichen Volumina $\mathrm{v}$ des Oeles und des Wassers enthaltenen Substanzmengen im Gleichgewicht der Vertheilung oder der Theilungscoëfficient ergiebt sich demnach als

2) Stschukarew, Ber. d. russ. phys.-chem. Ges. Bd. XXIX. 1897. (Chem. Centralbl. I. 1898. 1156.) 


$$
\mathrm{Q}=\frac{\mathrm{m}}{\mathrm{n}} \cdot \frac{\mathrm{a}-\mathrm{b}}{\mathrm{b}}(1)
$$

Setzen wir $p=\frac{m}{n}$

so wird $Q=p \cdot \frac{a-b}{b}$.

Werden gleiche Volumina Wasser und Oel angewendet, so ergiebt sich, da dann

$$
\begin{aligned}
& \mathrm{p}=1 \\
& \mathrm{Q}_{0}=\frac{\mathrm{a}_{0}-\mathrm{b}_{0}}{\mathrm{~b}_{0}}(2) .
\end{aligned}
$$

Nach dieser Gleichung gestalten sich die Versuche sehr einfach. Ein bestimmtes Volum einer wässerigen Lösung wird mit dem gleichen Volumen Oel erschöpfend durchgeschüttelt, und hierauf in einem aliquoten Theil derselben der Gehalt nach dem Schütteln bestimmt. Die Differenz des letzteren gegenüber dem ursprünglichen Werthe giebt die in dasselbe Volumen Oel übergegangene Substanzmenge an. Das Verhältniss dieses zu dem im Wasser verbliebenen Rest ist der Theilungscoëfficient. Zur Controle dieses Werthes und zur Prüfung, ob nicht die Concentration einen Einfluss auf die Vertheilung besitzt, empfiehlt es sich, Versuche nach Gleichung (1) mit verschiedenen Verhältnissen der wässerigen Lösung und des Oeles zu einander anzustellen. Es ist dies dem Verdünnen der ursprünglichen Lösung vorzuziehen, da in letzterem Falle das genaue analytische Arbeiten schwieriger ist. Aber auch die erstere Methode schliesst Bedenken in sich, da durch den Factor $p$ Versuchsfehler multiplicirt werden, ferner bei starkem Ueberwiegen des einen Lösungsmittels eine völlig: gleichmässige Durchsohtittelung beider mitunter fraglich ist. Ueberhaupt ergeben sich für $Q$ nach der Natur seiner Abhängigkeit viel grössere Differenzen, als für die beobachteten Werthe von b. Unter diesen Umständen ist es schwer, bei den verschiedenartig angestellten Versuchen übereinstimmende Werthe zu erhalten, und genau den Einfluss der Concentration zu ermitteln. Es wurden deshalb für die bereits im Vorausgehenden zusammengestellten Werthe nur diejenigen gewählt, welche nach der Versuchsanordnung gemäss der Gleichnng (2) erhalten wurden.

Die Ausführung bietet versehiedene Schwierigkeiten, die einmal in der Natur des Oeles gelegen sind, ferner darin, dass für jede einzelne Substanz eine geeignete Methode zur quantitativen Bestimmung des Gehaltes ihrer wässerigen Lösung ermittelt und geprüft werden muss. Letzteres geschah im Folgenden stets durch mehrfache Pa- 
rallelbestimmungen, zu welchen dasselbe Quantum der ursprünglichen Lösung, das nach dem Schütteln zur Analyse gelangen sollte, verwendet wurde. Man bedient sich dabei zweckmässig zu beiden Abmessungen derselben Messpipette, deren Volumen zweckentsprechend zu wählen ist. Die eigentlichen Vertheilungsversuche wurden ebenfalls in einer grösseren Zahl gut übereinstimmender Parallelbestimmungen ausgeführt.

Das 0 el. Zur Verwendung kam reinstes Olivenöl, von dem von vornherein ein grösseres Quantum derselben Provenienz bereit gestellt wurde. Vor dem Gebrauche wurde es längere Zeit mit Wasserdampf erhitzt, um alle ins Wasser übergehenden Bestandtheile, die beim Durehsehütteln eine starke milchige Trübung bewirken, zu entfernen. Vollständig gelang dies nicht, doch überzeugte ich mich, dass die mit Rücksicht darauf gewählten Bestimmungsmethoden dadurch nicht beeinträchtigt wurden.

Das Abmessen. Das abzumessende Volumen richtet sich in jedem Falle nach der für die darauffolgende Bestimmung erforderlichen Menge, indem man vorsieht, dass man bei der nachherigen Trennung der beiden Lösungen das gewünschte Volumen zur Verfiugung hat. Zum Abmessen dienen zweckmässig zwei $50 \mathrm{ccm}$ Büretten, die eine für die wässerigen Lösungen, die andere für das Oel. Das Ausfliessen des letzteren muss, um grobe Fehler zum vermeiden, ganz langsam tropfenweise erfolgen und nimmt deshalb viel Zeit in Anspruch. Als Schüttelgefässe werden je nach dem Volum gewöhnliche Reagensgläschen, besser noch dickwandige Centrifugircylinder, die mit weichgepressten Korken verschlossen werden, verwendet.

Das Sch ütteln erfolgt in einem immer gleichtemperirten Raume mittels einer Schüttelmaschine. Das Durchschütteln soll kräftig sein, da bei blossem Schwenken auch nach langer Zeit der Eintritt des Gleichgewichtes ausbleiben kann. Darauf hat man naturlich, um richtige Resultate zu erhalten, immer sein Augenmerk zu richten. In einem speciellen Falle überzengte ich mich, dass bei kräftigem Durchschütteln schon nach noch nicht 10 Minuten die Vertheilung dieselbe war, wie beim Fortsetzen des Schüttelns durch weitere zwei Stunden. Immerhin ist es angezeigt, die Dauer des Schüttelns genügend auszudehnen.

Trennung der beiden Lösungen. Dieselbe kann mannigfache Schwierigkeiten bieten. Aus nicht aufgeklärten Gründen erfolgt die Sonderung der beiden Schichten mitunter rasch und vollständig, während oft bei gleichzeitig unter denselben Umständen 
angestellten Versuchen die wässerige Lösung stark getrübt bleibt, und die Scheidung nur langsam und unvollkommen von statten geht. Als ein einfaches, recht brauchbares Mittel, die wässerige Lösung vom Oel zu befreien, erwiess sich das Filtriren durch ein zwei- bis dreifaches gewöhnliches Rundfilter. Man giesst die beiden Lösungen zusammen auf das trockene Filter und lässt das Filtrat in ein trockenes Gefäss fliessen. Dabei wird das Oel von dem nunmehr befeuchteten Filter zunächst zurückgehalten. Das Filtrat ist klar oder doch stark geklärt und ist von suspendirtem Oel befreit.

Gehalt der wässerigen Lösung nach dem Schütteln. Die Bestimmung desselben ist die eigentliche Hauptaufgabe im ganzen Versuche. Man bedient sich vortheilhaft zur Abmessung derselben Pipette, für welche man den Gehalt der ursprünglichen Lösung ebenfalls bestimmt hat. Eine vorhandene Trübung ist, wie schon erwähnt, belanglos. Bei der Prüfung der verschiedenen angewandten Methoden an sonst reinem Wasser, das durch Schütteln mit Oel die erwähnte Trübung aufwies, zeigte sich kein oder ein kaum merklicher Einfluss auf Gefrierpunkt, specifisches Gewicht, Titer einer zugesetzten Alkalimenge, und durch Ausäthern wurde die Trübung nicht beseitigt. Letzteres lässt sich übriges in vollkommenster Weise durch Erzeugung eines indifferenten Niederschlages, etwa von Baryumphosphat, erzielen, complicirt jedoch die Ausführung der Bestimmung beträchtlich.

Wegen der zumeist erfolgenden Verflüchtigung und der mitunter auch möglichen Zersetzung konnte im Folgenden die einfachste Methode, Verdampfung der wässerigen Lösung, überbaupt nicht zur Anwendung kommen. Je nach den chemischen Eigenschaften und den Löslichkeitsverhältnissen der verschiedenen Substanzen wurde der Gehalt der wässerigen Lösung bestimmt:

1. Durch Ausätherung bei der Gruppe der Sulfone. Sie sind sehwer löslich, und es muss deshalb ein grösseres Volumen zur Bestimmung genommen werden. Das Eindampfen desselben müsste sehr vorsichtig erfolgen, da bei raschem Verdampfen Verlust durch Verflüchtigung einträte. Bei anderen Substanzen lässt diese Methode schon wegen der Flüchtigkeit der festen Verbindung und der daraus erwachsenden Schwierigkeiten der Trocknung der ausgeätherten Menge im Stich.

2. Durch Ermittelung der Gefrierpunktsdepression: bei Aethylurethan. Der Vortheil dieser, sowie der folgenden Methode besteht darin, dass ein willkürliches Volumen zur Bestimmung verwandt werden kann. Wo jedoch eine chemische Methode unter 
Verwendung eines weit geringeren Flüssigkeitsquantums möglich war, wurde des weiteren dieser der Vorzug gegeben.

3. Durch das specifische Gewieht: bei Trimethylcarbinol (tertiärem Butylalkohol) und Dimethyläthylearbinol (tert. Amylalkohol, Amylenhydrat). Nur bei genügend concentrirten Lösungen erhält man Resultate von hinreichender Sicherheit, die auf empirisch gewonnenen Werten basiren müssen.

4. Durch Stickstoffbestimmung nach Kjeldahl: bei Methylurethan.

5. Durch Verseifung: bei den Acetinen und Chloralen.

\section{Die Sulfone.}

Die quantitative Bestimmung dieser Substanzen bietet eine beträchtliche Schwierigkeit insofern, als wegen der geringen Löslichkeit nur geringe Substanzmengen zur Bestimmung gelangen und daher möglichst genau gewogen werden müssen. Dies wird aber durch die Wägung in grösseren Glasgefässen sehr erschwert. Nach vielen Versuchen blieb ich bei dem Verfahren, dass ich das verschlossene Wägeglas aus dem Exsiccator eine Stunde vor der Wägung in die Wage stellte. Die Schwankungen bei den einzelnen Wägungen betrugen hierbei gegen $1 \mathrm{mg}$, so dass im ganzen Wägungsdifferenzen bis $\mathrm{zu} 2 \mathrm{mg}$ sich ergeben konnten, was oft mehrere Procente des absoluten Betrags ausmachte. Die Isolirung der Substanz aus der wässerigen Lösung: geschah aus den bereits angeführten Gründen durch Ausäthern mittels eines Extraktionsapparats. Die Dauer wurde zumeist auf $3-5$ Stunden erstreckt, obzwar wahrscheinlich auch eine kürzere Zeit genügt hätte.

Zunächst stellte ich mehrere Parallelversuche mit einer bestimmten Sulfonallösung an, für welche ich in $70,0 \mathrm{cem}$ die Mengen $0,0984-0,0999$ und $0,0996 \mathrm{~g}$, sowie in 20,0 ecm $0,0275-0,0254$ und $0,0290 \mathrm{~g}$ fand. Da ich nicht völlig identische Resultate erhalten konnte, wählte ich in solchen Fällen, wo die Uebereinstimmung für den Werth der Stammlösung in mehreren Controlbestimmungen nicht genug befriedigend erschien, für denselben den aus der angewandten Substanzmenge berechneten Titer. Von zahlreichen Versuchen zur Erzielung gut übereinstimmender Resultate gebe ich im folgenden die zuverlässigsten mit den daraus berechneten Theilungscoëfficienten wieder.

1. Sulfonal. Es wurde eine Lösung von $1,3856 \mathrm{~g}$ in $1000,0 \mathrm{ccm}$ angewandt, $50,0 \mathrm{ccm}$ mit $0,0693 \mathrm{~g}$ zeigten nach dem Schütteln mit dem gleichen Volum Oel einen Gehalt von $0,0350 \mathrm{~g}$ nach dem Schütteln mit 
$25,0 \mathrm{ccm}$ Oel verblieben im Wasser $0,0420 \mathrm{~g}$. Daraus berechnen sich die Theilungscoëfficienten

$$
\left.\begin{array}{l}
\mathrm{Q}_{1}=\frac{1}{1} \cdot \frac{693-350}{350}=0,979 \\
\mathrm{Q}_{2}=\frac{2}{1} \cdot \frac{693-420}{420}=1,251
\end{array}\right\} \text { Mittel : 1,115. }
$$

2. Trional. Es wurden $1,0069 \mathrm{~g}$ in $500,0 \mathrm{ccm}$ gelöst. Aus $50,0 \mathrm{cem}$ wurden 0,1027 und $0,1024 \mathrm{~g}$, im Mittel $0,1025 \mathrm{~g}$ ausgeäthert. Der Gehalt derselben betrug nach dem Schïtteln mit dem gleichen Volum Oel $0,0202 \mathrm{~g}$, mit dem halben Volum Oel $0,0300 \mathrm{~g}$, und darans berechneten sich die Theilungscoëfficienten

$$
\left.\begin{array}{l}
\mathrm{Q}_{1}=\frac{1}{1} \cdot \frac{1025-202}{202}=4,074 \\
\mathrm{Q}_{2}=\frac{2}{1} \cdot \frac{1025-300}{300}=4,833
\end{array}\right\} \text { Mittel: 4,458. }
$$

3. Tetronal. Es wurden $1,7661 \mathrm{~g}$ in $750,0 \mathrm{ccm}$ gelöst, schieden sich jedoch beim Abkühlen zu einem geringen Theil wieder ans. Beim Ansäthern fanden sich $0,0954 \mathrm{~g}$ in 50,0 ecm. Nach dem Schütteln der wässerigen Lösung mit Oel im Verhältniss der ersteren zum letzteren wie $6: 5$, resp. $16: 9$ fanden sich in $50,0 \mathrm{ccm} 0,0231 \mathrm{~g}$, resp. $0,0276 \mathrm{~g}$. Daraus berechnen sich die Theilungscoëfficienten

$$
\left.\begin{array}{l}
\mathrm{Q}_{1}=\frac{6}{5} \cdot \frac{954-231}{231}=3,756 \\
\mathrm{Q}_{2}=\frac{16}{9} \cdot \frac{954-276}{276}=4,322
\end{array}\right\} \text { Mittel: } \mathbf{4 , 0 3 9} .
$$

4. Dimethylsulfondimethylmethan. $1,6737 \mathrm{~g}$ warden in $250,0 \mathrm{ccm}$ gelöst, auf 50,0 ecm kamen also $0,3347 \mathrm{~g}$. Nach dem Schütteln mit dem gleichen Volum Oel fanden sich in demselben $0,3036 \mathrm{~g}$, in der anf die Hälfte verdünnten Lösung ebenfalls nach dem Schütteln mit dem gleichen Volum Oel $0,1509 \mathrm{~g}$. Daraus berechnen sich die Theilungscoëfficienten

$$
\left.\begin{array}{l}
\mathrm{Q}_{1}=\frac{1}{1} \cdot \frac{3347-3036}{3036}=0,1028 \\
\mathrm{Q}_{2}=\frac{2}{1} \cdot \frac{1674-1509}{1509}=0,1091
\end{array}\right\} \text { Mittel: } \mathbf{0 , 1 0 5 9 .}
$$

5. Diäthylsulfonmethan. $0,3514 \mathrm{~g}$ wurden in $50,5 \mathrm{ccm}$ gelöst, $42,0 \mathrm{ccm}$ mit dem gleichen Volum Oel 4 Stunden geschüttelt und sodann $36,9 \mathrm{ccm}$ ausgeäthert. Dieselben enthielten noch $0,2252 \mathrm{~g}$, ihr ursprünglicher Gehalt berechnet sich zu $0,2593 \mathrm{~g}$ und daraus der Theilungscoëfficient

$$
\mathrm{Q}=\frac{2593-2253}{2253}=0,1514 \text {. }
$$

\section{Aethyl- und Methylurethan.}

Für das erstere wurde der Gebalt der wässerigen Lösung dureh Bestimmung ihrer Gefrierpunktsdepression ermittelt, für letzteres durch Stickstoffbestimmung nach $\mathrm{Kj}$ eldahl. 
6. Das Aethylurethan. $1,1895 \mathrm{~g}$ in $18,0 \mathrm{ccm} \mathrm{H}_{2} \mathrm{C}$ zeigten eine Gefrierpunktsdepression $\Delta=1,291^{\circ}$, woraus sich ein Gehalt von 6,212 Proc. (angewandt 6,199 Proc.) ergiebt. Ferner zeigten 0,240 in $18,0 \mathrm{cem}$ $\Delta=0,268^{\circ}$ (berechnet $0,274^{\circ}$ ).

Zur Bestimmung des Theilungscoëfficienten wurde eine Lösung, die $\Delta=0,909^{\circ}$ (berechnet $0,915^{\circ}$ ) zeigte, mit dem gleichen Volum Oel geschüttelt, und besass hierauf im Mittel zweier Versuche $A=0,800^{\circ}$, daher

$$
\mathrm{Q}=\frac{1}{1} \frac{909-800}{800}=\mathbf{0 , 1 3 6}
$$

Nach dem Schütteln von 5 Theilen der wässerigen Lösung mit 6 Theilen Oel zeigte erstere $A=0,780$, daher

$$
\mathrm{Q}=\frac{5}{6} \cdot \frac{909-780}{780}=0,13 \%
$$

7. Methylurethan. $3,75 \mathrm{~g}$ wurden in $50,0 \mathrm{ccm}$ zu einer $1 / 1 \mathrm{n}$. Lösung gelöst. $5 \mathrm{ccm}$ derselben verbrauchten bei der Bestimmung des Stickstoffs nach $\mathrm{Kj}$ eldahl für den gebildeten Ammoniak eine Säuremenge, die 49,55 und $49,82 \mathrm{ccm}{ }^{1} / 10 \mathrm{n}$. KOH äquivalent war statt berechneten $50,0 \mathrm{ecm}$. Nach dem Schütteln gleicher Volumina Lösung und Oel verbrauchten $5 \mathrm{ccm}$ der ersteren 47,91 und $46,06 \mathrm{~cm} \mathrm{KOH}$. Letzterer Werth muss jedoch wegen gewisser Unregelmässigkeiten bei der Bestimmung ausgeschaltet werden, so dass sich berechnet

$$
\mathrm{Q}=\frac{1}{1} \frac{49,68-47,91}{47,91}=\mathbf{0 , 0 3 6 \%} \text {. }
$$

\section{Tertiärer Butyl- und Amylalkohol.}

Die Bestimmung des Gehaltes erfolgte durch specifisches Gewicht. Diese Methode gestaltete 'sich einfach derart, dass zunäehst das Gewicht eines gläsernen Senkkörpers in der Stammlösung und in versehieden abgestuften Concentrationen derselben bei constanter Temperatur bestimmt und hierauf aus seinem Gewicht in der geschüttelten Lösung bei derselben Temperatur deren Gehalt berechnet wurde. Der cylindrische Senkkörper war nach Kohlrausch mittels einer eingeschmolzenen Platinöse mit einem feinen Frauenhaar verknüpft, dessen oberes Ende mit einem Platindraht zum Aufhängen an den Wagebalken versehen war.

8. Tertiärer Butylalkohol. $4,3721 \mathrm{~g}$ wurden in $50,0 \mathrm{ccm}$ gelöst. Nach dem Schütteln mit dem gleichen Volumen Oel betrug das Gewicht des Glaskörpers in der geschüttelten Lösung 8,9941 $\mathrm{g}$ und war identisch mit dem Gewichte in derjenigen Lösung, welche durch Vermischen von 8,5 Theilen der Stammlösung mit 1,5 Theilen Wasser erhalten wurde. Daher ergiebt sich

$$
\mathrm{Q}=\frac{1,5}{8,5}=\mathbf{0 , 1 7 6 4}
$$


9. Amylenhydrat. $13,2118 \mathrm{~g}$ wurden in $200,0 \mathrm{cem}$ gelöst. Es ergab sich zunächst

\begin{tabular}{c|c}
\hline $\begin{array}{c}\text { Gehalt der } \\
\text { Lösung } \\
\text { in } 1 \mathrm{ccm}\end{array}$ & $\begin{array}{c}\text { Gewicht des } \\
\text { Glaskörpers }\end{array}$ \\
\hline $66,1 \mathrm{mg}$ & $8,9807 \mathrm{~g}$ \\
$44,1=$ & $8,9638=$ \\
$33,0=$ & $8,9574=$ \\
$0,00=$ & $8,9306=$
\end{tabular}

Nach zweistündigem Schütteln der Stammlösung mit dem gleichen Volum Oel zeigte der Glaskörper in der geschüttelten Lösung das Gewicht $8,9577 \mathrm{~g}$ und nach weiterem vierstïndigen Schütteln $8,9574 \mathrm{~g}$, entsprechend $33,0 \mathrm{mg}$ in $1 \mathrm{ccm}$. Darans ergiebt sich der Theilnngscoëfficient

$$
Q=\frac{66,1-33,0}{33,0}=1,000 \text {. }
$$

Auch der Theilungscoëfficient des Chloralhydrats, von dem noch später die Rede sein wird, wurde nach dieser Methode bestimmt. Dabei ergab sich:

\begin{tabular}{c|c}
\begin{tabular}{c} 
Gehalt der $\begin{array}{c}\text { Lösung } \\
\text { in } 1 \mathrm{ccm}\end{array}$ \\
\hline $0,1631 \mathrm{~g}(1 / 1)$
\end{tabular} & $\begin{array}{c}\text { Gewicht des } \\
\text { Glaskörpers }\end{array}$ \\
\hline $0,1360=(1 / 6)$ & $8,5030 \mathrm{~g}$ \\
$0,1223=(1 / 4)$ & $8,6470=$ \\
$0,0815=(1 / 2)$ & $8,7172=$ \\
$0,0326=(1 / 5)$ & $8,8597=$ \\
$0,0000=$ & $8,9302=$
\end{tabular}

Zur Bestimmung von $\mathbf{Q}$ wurde die Stammlösung sowie die anf die Hälfte verdünnte Lösung mit dem gleichen Volumen Oel geschüttelt.

\begin{tabular}{|c|c|c|}
\hline $\begin{array}{l}\text { Gehalt } \\
\text { der Lösung: } \\
\text { vorher }\end{array}$ & $\begin{array}{l}\text { Gewicht des } \\
\text { Schwimmkörpers in } \\
\text { geschüttelter Lösung }\end{array}$ & $\begin{array}{l}\text { Gehalt der } \\
\text { geschüttelten } \\
\text { Lösung }\end{array}$ \\
\hline $0,1631 \mathrm{~g}$. & $8,5730 \mathrm{~g}$ & 0,1370 \\
\hline $0,0815=$ & $8,7570=$ & 0,0670 \\
\hline
\end{tabular}

Daraus ergeben sich

$$
\begin{aligned}
& \mathrm{Q}_{1}=\frac{1631-1370}{1370}=\mathbf{0 , 1 9 0 4} \\
& \mathrm{Q}_{2}=\frac{815-670}{670}=\mathbf{0 , 2 1 6 4 .}
\end{aligned}
$$

\section{Acetine.}

Die Bestimmung des Mono-, Di- und Triacetins erfolgte durch Verseifung mit $1 / 10$ n.-Lauge. Controlbestimmungen ergaben, dass ein 
geringer Uebersehuss der Lauge und einstündiges Erwărmen auf dem Wasserbade, selbst ebenso langes Stehen bei Zimmertemperatur zur vollständigen Verseifung hinreichen.

10. Monoacetin, $\mathrm{C}_{3} \mathrm{H}_{5}(\mathrm{OH})_{2}$. $\left(\mathrm{OC}_{2} \mathrm{H}_{3} \mathrm{O}\right)$. Das angewandte Präparat wurde im Vacuum fractionirt and ging grösstentheils zwischen $169-170^{\circ}$ bei $28 \mathrm{~mm}$ über. Es gelangte eine Lösung mit $0,0428 \mathrm{~g}$ in $1 \mathrm{cem}$ zur Verwendung und verbrauchte $3,880 \mathrm{~cm} 1 / 10 \mathrm{n}$. Lauge für dieselben. Nach dem Schütteln gleicher Volumina Lösung und Oel verbranchte $1 \mathrm{ccm}$ wässeriger Lösung in 2 Controlbestimmungen 3,682 , resp. $3,645 \mathrm{ccm} 1 / 10 \mathrm{n} . \mathrm{KOH}$, im Mittel $3,664 \mathrm{ccm}$. Daraus ergiebt sich

$$
\mathrm{Q}=\frac{1}{1} \cdot \frac{3,880-3,664}{3,664}=0,0593 \text {. }
$$

11. Diacetin, $\mathrm{C}_{3} \mathrm{H}_{5}(\mathrm{OH})\left(\mathrm{OO}_{2} \mathrm{H}_{3} \mathrm{O}\right)_{2}$. Das Präparat ging bei $160^{\circ}$ unter $25 \mathrm{~mm}$ Druck ỉber. $5 \mathrm{ccm}$ der ursprünglichen und der mit dem gleichen Volumen Oel geschuittelten Lösung verbrauchten $1 / 10 \mathrm{n} . \mathrm{KOH} 17,73 \mathrm{ccm}$, resp. 14,37 ccm; daher

$$
Q=\frac{1}{1} \cdot \frac{1773-1437}{1437}=0,234 .
$$

12. Triacetin, $\mathrm{C}_{3} \mathrm{H}_{5}\left(\mathrm{OC}_{2} \mathrm{H}_{3} \mathrm{O}\right)_{3}$. Siedete unter $31 \mathrm{~mm}$ Druck bei $167^{0}$. Eine Lösung mit $0,0272 \mathrm{~g}$ in $1 \mathrm{ccm}$ verbranchte für $5,0 \mathrm{~cm}$ vor und nach dem Schütteln mit dem gleichen Volum $0 e 1$ 15,1 $\mathrm{cem}$ und $11,66 \mathrm{ccm}$ $1 / 10 \mathrm{n} . \mathrm{KOH}$, daher

$$
\mathrm{Q}=\frac{1}{1} \cdot \frac{15,1-11,66}{11,66}=\mathbf{0 , 2 9 5 1 .}
$$

\section{Chlorale.}

Das Chloralhydrat, $\mathrm{CCl}_{3}$. $\mathrm{CH}(\mathrm{OH})_{2}$, Bromalhydrat, $\mathrm{CBr}_{3} . \mathrm{CH}(\mathrm{OH})_{2}$, und Butyehloralhydrat, $\mathrm{O}_{3} \mathrm{H}_{4} \mathrm{Cl}_{3} \mathrm{CH}(\mathrm{OH})_{2}$, werden bereits durch Einwirkung von $1 / 10 \mathrm{n}$. Kalilauge auf ihre wässerige Lösung gespalten. Die beiden ersten zerfallen dabei in Ameisensäure und Chloroform, bezw. Bromoform nach dem Schema

$$
\mathrm{CX}_{3} \cdot \mathrm{CHO}+\mathrm{H}_{2} \mathrm{O}=\mathrm{CX}_{3} \mathrm{O}+\mathrm{H} . \mathrm{COOH} \text { (1). }
$$

Die Zersetzung des Butylohloralhydrats entspricht der Gleichung

$$
\begin{aligned}
& \mathrm{C}_{4} \mathrm{H}_{5} \mathrm{Cl}_{3} \mathrm{O}+\mathrm{H}_{2} \mathrm{O}=\mathrm{C}_{3} \mathrm{H}_{4} \mathrm{Cl}_{2}+\mathrm{HCl}+\mathrm{CH}_{2} \mathrm{O}_{2} \text { (2) } \\
& \text { Butylchloral Dichlorpropylen Ameisensäure }
\end{aligned}
$$

Gleichung (1) erfordert ein, (2) zwei Aequivalente Lange. Das Butylchloralhydrat verbrauchte ron derselben genau die theoretische Menge, dagegen findet beim Chloral- und Bromalhydrat selbst durch die verdiunnte Lauge sehr leicht eine theilweise Zersetzung des abgespaltenen Halogenkohlenwasserstoffes statt. Dies suchte ich durch bloss einstiundiges Verseifen bei Zimmertemperatur zu vermeiden und erhielt unter dieser Bedingung ziemlich übereinstimmende Resultate, die aber bereits einem geringen Mehrverbrauch an Lauge entsprachen. Derselbe wuchs durch Ausdehnung der Verseifungsdauer ansehnlich, 
und es sollte deshalb durch Zurücktitriren der Lauge mit Essigsäure und Bestimmung des gebildeten Chlorids mit $\mathrm{AgNO}_{3}$ nach Voll$\mathrm{h}$ ard t der Fehler eliminirt werden, der aus der weitergehenden Zersetzung stammte. Nach älteren Angaben würde diese für das Chloroform nach der Gleichung:

$$
\mathrm{CHCl}_{3}+4 \mathrm{KOH}=\mathrm{HCOOK}+3 \mathrm{KCl}+2 \mathrm{H}_{2} \mathrm{O}
$$

erfolgen, und so wären demnach $4 / 3$ der der gefundenen Salzsäure äquivalenten Menge $\mathrm{KOH}$ von der insgesammt verbrauchten abzuziehen. Die nach dieser Ueberlegung angestellten Versuche zeigten zunächst noch weniger eine Uebereinstimmung, und es wurde daher bei Ermittelung der Theilungscoëfficienten der Gehalt der Chloraland Bromallösung einfach durch einstündiges Verseifen bei Zimmertemperatur bestimmt, da unter Einhaltung dieser Bedingung ziemlich ibereinstimmende Werthe erhalten werden, die ron dem theoretisch geforderten nur unbedeutend differiren.

13. Für das Chloralhydrat stellte ich Mischungsversuche mit verschiedenen Verhältnissen von Oel zu Wasser an, wobei für jedes Verhältniss 2 Controlversuche ausgeführt und jede der geschüttelten wässerigen Lösungen in 2 Portionen analysirt wurden.

Die Stammlösung des Chloralhydrates enthielt in $1 \mathrm{ccm} 0,0412 \mathrm{~g}$; von der Lauge entsprach $1 \mathrm{ccm} 0,003568 \mathrm{~g} \mathrm{HCl}$ und sollte nach Gleichung (1) $0,01612 \mathrm{~g}$ Chloralhydrat äquivalent sein. Demnach sollte $1 \mathrm{ccm}$ ersterer Lösung $2,558 \mathrm{ccm}$ der Lange verbrauchen. Ich fand, dass nach einstündigem Verseifen bei Zimmertemperatur 12,0 cem Lösung 31,62 und $31,65 \mathrm{~cm} \mathrm{KOH}$ verbraucht hatten, im Mittel für $1 \mathrm{ccm} 2,636 \mathrm{ccm}$ Lauge.

Die bei der Bestimmung des Theilungscoëfficienten erhaltenen Resultate sind in folgender Tabelle zusammengestellt.

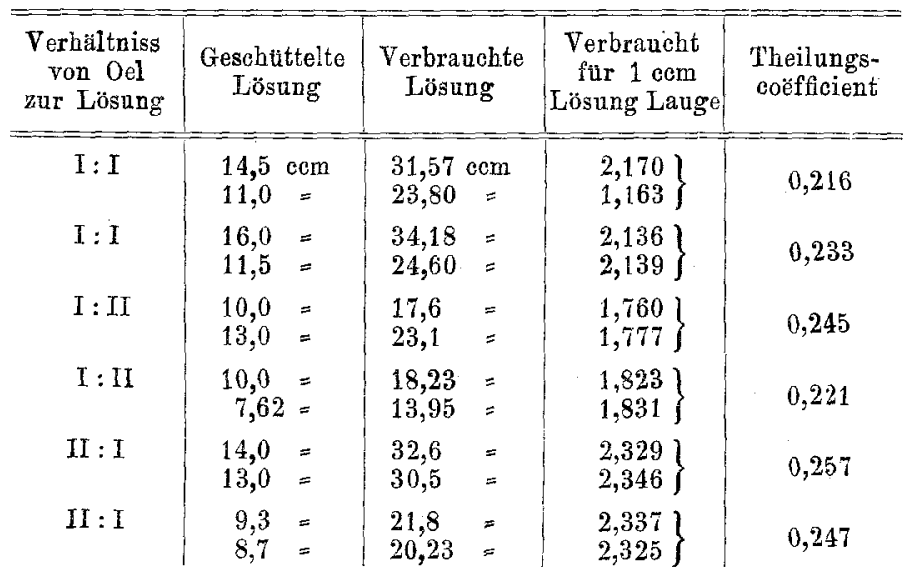

Die Ungenauigkeit der Methoden gestattet es leider nicht, ans diesen Werthen einen sicheren Schluss auf einen etwa vorhandenen Einfluss der 
Concentration auf die Vertheilung zu ziehen. In Anbetracht jener Möglichkeit ist es aber auch unzulässig, aus ihnen einen Mittelwerth zn bilden.

Deshalb wurde in die schon frïher gegebene Zusammenstellung als Theilungscoëfficient etwas willkürlich der Werth 0,216 aufgenommen, der übrigens mit dem durch Bestimmnng des specifischen Gewichtes erhaltenen Werthe $Q_{2}$ (siehe $S .133$ ) sehr gut ibbereinstimmt.

Durch die mir später im Anszug bekannt gewordene Arbeit von A. Desgrez ${ }^{1}$ ) über die Zersetzung von $\mathrm{CHCl}_{3}$ u. s. w. durch wässerige Kalilauge gelang es mir ïbrigens nachträglich, die verwickelten Verhältnisse bei der Verseifung des Chloralhydrates zu durchblicken und damit die Bedingungen für die Erzielung richtiger Werthe anfaufinden. Nach jenem Autor erfolgt die Zersetzung des Chloroforms, besonders leicht bei der Abspaltung aus Chloral, nach der Gleichung

$$
\mathrm{CHCl}_{3}+\mathrm{KOH}=\mathrm{CO}+\mathrm{KCl}+2 \mathrm{HCl} \text {. }
$$

Demnach ist der Mehrverbranch an Lauge gerade der gefundenen $\mathrm{HCl}$ äquivalent. Unter Zngrundelegung dieses Verhältnisses ergab die Unrechnung der früher exhaltenen Versuchsresultate, dass durch einstündiges Verseifen bei Zimmertemperatur die Verseifung überhaupt noch nicht vollständig erfolgt ist, aber ein Theil der KOH, deren Gesammtverbrauch nahezu die theoretisch geforderte Menge betrug, bereits an Salzsäure gebunden war. Dagegen erhielt ich bei jenen Versuchen, bei welchen die Verseifung entweder mehrere Stunden gedauert oder unter Erwärmung auf dem Wasserbad stattgefunden hatte, jetzt sehr gut stimmende Resultate, obwohl im letzteren Fall sich beträchtliche Mengen Chlorid gebildet hatten. Es ergiebt sich das Gesagte aus der folgenden Zinsammenstellung.

\begin{tabular}{|c|c|c|c|c|c|}
\hline $\begin{array}{l}\text { Chloral- } \\
\text { lossung }\end{array}$ & Verseifung & Gesammt $\mathrm{KOH}$ & $\begin{array}{c}\text { Dem Chlorid } \\
\text { aquivalente } \\
\mathrm{KOH}\end{array}$ & $\begin{array}{r}1 \text { ccm C } \\
\text { verbral } \\
\text { uncorr. } \\
\text { a. } \\
\end{array}$ & $\begin{array}{c}\text { ralhydrat } \\
\text { KOH } \\
\text { corrig. } \\
\text { b. }\end{array}$ \\
\hline $15,0 \mathrm{ccm}$ & $1 \mathrm{~h}$. Zimmertem $\mathrm{p}$. & $\begin{array}{l}\frac{38,48}{38,45} \\
\frac{38,46}{\text { Nittel }}\end{array}$ & - & $\frac{-}{2,564}$ & - \\
\hline $21,0=$ & & 53,9 & $0,83 \mathrm{ccm}$ & 2,567 & 2,527 \\
\hline $12,0=$ & $1 \mathrm{~h}$. Zimmertemp. & 30,74 & $0,32=$ & 2,562 & 2,535 \\
\hline $10,0=$ & & 25,61 & $0,30=$ & 2,561 & 2,531 \\
\hline $15,0=$ & 9 h. Zimmertemp. & 38,76 & $0,5=$ & 2,584 & 2,551 \\
\hline $15,0=$ & $1^{1 / 2} \mathrm{~h}$. bei $70^{\circ}$ & 44,58 & $6,32=$ & 2,972 & 2,551 \\
\hline
\end{tabular}

Die zwei letzten Werthe der Columne b, 2,551, zeigen eine sehr gute Uebereinstimmung mit dem berechneten Werthe 2,558. Aber auch die vier ersten uncorrigirten (in Bezug auf das gebildete Chlorid) Werthe der Columne a weichen von demselben nicht nennenswerth ab, trotzdem die Zersetzung des Chloralhydrates noch nicht vollständig ist. Es können deshalb die unter Einhaltung bestimmter Bedingungen bloss durch Zurücktitriren der verbrauchten Lauge gewonnenen Werthe als verwendbar betrachtet werden. So verhält es sich anch beim

1) Desgrez, Compt. rendus T. CXXV. p. 780. 1898. 
14. Bromalhydrat. Nach Gleichung (1) entspricht $1 \mathrm{ccm} \mathrm{1/10} \mathrm{n.} \mathrm{KOH}$ $=0,02985 \mathrm{~g}$ Bromalhydrat. Von der verwendeten Lauge war $1 \mathrm{ccm}=$ $0,997 \mathrm{~cm} 1 / 10 \mathrm{n} . \mathrm{KOH}=0,02915 \mathrm{~g}$ Bromalhydrat. In 2 Versuchen ergab sich das eine Mal ein Verbranch von $1 \mathrm{ccm}$ der Lange

für $0,02957 \mathrm{~g}$ nach $1 / 4$ Stunde und $0,02742 \mathrm{~g}$ nach $16 \mathrm{~h}$,

für $0,02946 \mathrm{~g}$ nach 1 Stunde und $0,02821 \mathrm{~g}$ nach $4 \mathrm{~h}$.

in dem 2. Versuche. Die nach 1/4- und einstündiger Verseifung bei Zimmertemperatnr erhaltenen Werthe sind gut übereinstimmend und entsprechen nahezu dem berechneten Werthe.

Nach dieser Methode bestimmt, verbrauchten 5,0 ccm der Stammlösung 16,90 und 16,74, im Mittel 16,82 $\mathrm{ccm}$ Lauge, nach dem Schütteln mit dem gleichen Volumen Oel 10,05 - 10,21 und 10,03, im Mittel 10,10 cem Lauge; daher der Theilungscoëfficient

$$
\mathrm{Q}=\frac{1}{1} \frac{6,72}{10,10}=\mathbf{0 , 6 6 5}
$$

15. Das Butylchloralhydrat. Nach zweistündigem Stehen bei Zimmertemperatur und nachfolgendem kurzen Erwärmen verbrauchten 0,5873 g $52,87 \mathrm{ccm}$ und $0,4931 \mathrm{~g} 51,38 \mathrm{~cm} 1 / 10$ KOH. $1 \mathrm{ccm}$ derselben entsprach daher $0,009594 \mathrm{~g}$ und $0,009596 \mathrm{~g}$ Butylehloralhydrat, während theoretisch nach (2) $0,009671 \mathrm{~g}$ erfordert werden.

$10,0 \mathrm{ccm}$ einer Lösung von $2,0374 \mathrm{~g}$ in $100,0 \mathrm{ccm}$ verbrauchten im Mittel zweier Bestimmungen 19,26 ccm KOH. Nach dem Schütteln mit dem gleichen Volumen Oel 7,4 ecm, daher

$$
\mathrm{Q}=\frac{19,26-7,4}{7,4}=\mathbf{1 , 5 8 9} \text {. }
$$

November 1898. 